\section{EDUCACIÓN PARA EL DESARROLLO, CODESARROLLO Y EL PAPEL DE LAS PERSONAS MIGRANTES. UNA REFLEXIÓN DESDE LA ANTROPOLOGÍA A PARTIR DE UN CASO CONCRETO}

\author{
Alba Vallés Marugán \\ Universidad de Granada \\ ORCID ID: 0000-0003-2021-6370 \\ albavalles@ugr.es
}

Cómo citar este artículo/Citation: Vallés Marugán, Alba (2021). Educación para el Desarrollo, codesarrollo y el papel de las personas migrantes. Una reflexión desde la Antropología a partir de un caso concreto. Arbor, 197(799): a596. https://doi. org/10.3989/arbor.2021.799009

Recibido: 4 enero 2020. Aceptado: 13 mayo 2020.

Publicado: 9 abril 2021

RESUMEN: La presencia de migrantes provenientes de contextos diversos constituye un elemento definitorio de las plurales sociedades del norte global, por lo que se perciben como necesarias acciones que favorezcan la convivencia entre personas migrantes y autóctonas. En esta línea, una de las propuestas planteadas desde la Educación para el Desarrollo pasa por fomentar la integración mediante acciones que sensibilicen sobre el fenómeno migratorio y las experiencias de los y las migrantes, destacando proyectos en que estos asumen el rol de agentes de desarrollo, vinculando migraciones y desarrollo, del modo positivo planteado desde el codesarrollo.

A partir del análisis de un proyecto llevado a cabo en Granada, pretendemos reflexionar sobre cómo toma forma el codesarrollo en las sociedades receptoras, cuál es el papel de los y las migrantes en estos procesos y cómo puede la Antropología aportar una visión compleja y crítica sobre el codesarrollo y la Educación para el Desarrollo.

PALABRAS CLAVE: Educación para el Desarrollo, codesarrollo, migraciones, integración, Antropología.
EDUCATION FOR DEVELOPMENT, CO-DEVELOPMENT AND THE ROLE OF MIGRANTS. AN ANTHROPOLOGICAL REFLECTION BASED ON A SPECIFIC CASE

Copyright: (C) 2021 CSIC. Este es un artículo de acceso abierto distribuido bajo los términos de la licencia de uso y distribución Creative Commons Reconocimiento 4.0 Internacional (CC BY 4.0).

ABSTRACT: The presence of people from different cultural contexts is an element that defines current plural societies in the Global North. As such, applying actions that promote mutual coexistence between migrants and local people is perceived as being necessary. In this line, one of the suggestions from Development Education focuses on promoting integration through actions to raise awareness on the migration phenomenon and the experiences of migrant people. Among these initiatives, we highlight the projects in which the migrants assume a role as agents in Development Education, linking the migratory phenomenon and development, viewed positively when considered from the perspective of co-development.

Based on analysis of a project done in Granada, we will reflect on how co-development can be introduced into host societies and what the role of migrants is in these processes. In addition, we will reflect on how Anthropology can contribute with a complex and critical approach to all these phenomena.

KEYWORDS: Development Education, co-development, migrations, integration, Anthropology. 


\section{INTRODUCCIÓN}

Una de las principales consecuencias de la globalización ha venido siendo, en los últimos años, la intensificación del fenómeno migratorio (Lacomba y Cloquell, 2017), lo que ha supuesto que la presencia de personas provenientes de países y contextos culturalmente diferentes constituya un elemento definitorio de las plurales sociedades actuales. En este sentido, la presencia de migrantes extranjeros no hace más que incrementar la diversidad de las ya de por sí heterogéneas sociedades occidentales, siendo el fenómeno migratorio uno de los posibles contextos -que no el único- de la multiculturalidad -o, como propondremos más adelante, la interculturalidad- (Zapata, 2003). En el caso que nos ocupa, esta cuestión nos interesa en dos sentidos diferentes: por un lado, tanto a nivel político, en cuanto al diseño e implementación de políticas públicas; como social, debido a las consecuencias que puede tener la incorporación de personas extranjeras en la creación de discursos sobre el fenómeno migratorio y el devenir de la convivencia y el día a día de personas locales y extranjeras. En este sentido, según Zamora (2003: 166) «el fenómeno social de la inmigración puede ser visto como un catalizador de las dinámicas sociales, económicas, políticas y culturales que definen la situación de las sociedades consideradas desarrolladas». De este modo, podemos comprobar que el proceso migratorio no afecta solo a los propios migrantes, sino que puede conllevar cambios en las sociedades implicadas en el mismo, tanto emisoras como receptoras (Cloquell, 2013) e, incluso, en aquellas por las que transitan durante sus proyectos migratorios.

Por otro lado, se trata de un campo que interesa a las Ciencias Sociales, pues éstas ven en la otredad implícita del fenómeno migratorio una fuente de datos muy amplia acerca de la construcción de identidades y alteridades. Estos son dos de los temas que han venido siendo objeto de investigación por parte de la Antropología, disciplina de la que partimos como investigadoras, entendiéndola como ciencia social que estudia al ser humano en todas sus dimensiones y complejidades. Así, uno de los múltiples motivos por los cuales la Antropología se interesa por el fenómeno migratorio se refiere a que el contacto entre personas de diferentes lugares y contextos socioculturales propiciado por el mismo, puede conllevar un choque identitario y un replanteamiento y reconstrucción de las identidades, tanto de las personas migrantes como de las autóctonas (Vallés, 2017).
Además, cuando estos migrantes proceden de países del denominado sur global, podemos plantearnos nuevas cuestiones relacionadas con el papel que puede tener la Antropología en los procesos migratorios y en los contextos en que éstos se vinculan al fenómeno del desarrollo: ¿cómo puede la Antropología participar en estos procesos, no solo de investigación, sino también de construcción y/o deconstrucción de la categoría de otro que constituye el migrante, con todo lo que ello conlleva? ¿Por qué le interesa el vínculo existente entre migraciones y desarrollo y cómo se aproxima a uno de sus fenómenos resultantes, a saber, el codesarrollo? Finalmente, ¿cómo se interesa por la participación de las personas migrantes en las sociedades receptoras?

En el caso que nos ocupa, el otro que constituye el migrante no es diferente únicamente por provenir de un contexto sociocultural distinto, sino que, además, los flujos migratorios de los que forma parte se establecen entre polos opuestos del sistema mundial, tal y como es entendido a nivel económico: los y las migrantes proceden de países del sur global, también considerados en vías de desarrollo, y que en este contexto se erigen como principales emisores de flujos migratorios. En contrapartida, las sociedades receptoras de estos flujos constituirían el norte global, los países considerados desarrollados. Es en este sentido, precisamente, en que se hace relevante el vínculo entre migraciones y cooperación para el desarrollo (Faist, 2010): los países del norte, receptores de migrantes, se erigen también como donantes de ayuda para el desarrollo de los países del sur, emisores y a la vez beneficiarios de dichas contribuciones. Con todo ello, vemos cómo norte y sur globales ocupan una doble posición polarizada y polarizante ${ }^{1}$. Asimismo, este hecho favorece también que, desde la Antropología del desarrollo, se torne relevante el codesarrollo, en la medida en que ésta se interesa por temas candentes relacionados con el campo de reflexión y aplicación de una noción crítica del desarrollo (Escobar, 1999).

Pero, partiendo de este planteamiento, ¿de qué desarrollo estamos hablando y cómo se ve afectado por el fenómeno migratorio? En base a estos cuestionamientos, nuestro interés se aproxima a la forma en que la Antropología, en su estudio de las migraciones, incorpora la cuestión del desarrollo y de las relaciones entre Estados en base a la cooperación internacional. Asimismo, reflexionamos también acerca de cómo el inmigrante, ese otro del que hablamos anteriormen-

1 Resulta interesante plantearse en qué sentido, desde la propia investigación antropológica se construyen, mantienen o legitiman estos discursos polarizantes. Para un acercamiento a la postura crítica de los estudios decoloniales sobre la construcción del sistema hegemónico mundial, consultar a Grosfoguel (2006), entre otros. 
te, participa y se ve afectado por los procesos de desarrollo. Pero, ¿de qué forma la hace respecto a su sociedad de origen? $Y$, también, ¿qué tipo de prácticas implementa en la sociedad receptora? ¿Son éstas consideradas acciones de desarrollo o, como planteamos aquí, de codesarrollo? ¿Puede el fenómeno migratorio beneficiar también los procesos de integración?

El codesarrollo surge, así, como una nueva problemática en nuestra investigación, en el sentido de que vincula ambos fenómenos, migración y desarrollo, teniendo como punto de partida la implicación y acciones puestas en práctica por los propios migrantes (Lacomba, 2010). En este sentido, Lotero-Echeverri y Pérez-Rodríguez (2019), al hacer un repaso al estado de la cuestión de las migraciones contemporáneas, resaltan el vínculo entre migraciones y desarrollo y su canalización bajo la forma del codesarrollo precisamente por la participación activa de las personas migrantes, pese a que también se refiere a la posible dependencia que se crea entre ambos fenómenos y por parte de la comunidad de origen hacia las personas migradas. Sin embargo, como veremos más adelante, la perspectiva del codesarrollo sigue teniendo relevancia en tanto en cuanto el vínculo entre migraciones y desarrollo sigue ocupando un lugar relevante en los debates políticos migratorios.

Así, en este texto nos gustaría seguir alimentando la reflexión sobre cómo puede tomar forma el codesarrollo en las sociedades receptoras, barajando como posible opción la Educación para el Desarrollo, así como el rol que puede jugar la Antropología en el análisis de ambos. Para ello, tras plantear ambas nociones, nos centraremos en el papel jugado por quienes migran, que parecen erigirse aquí como agentes de desarrollo, a partir del análisis de un proyecto concreto llevado a cabo en la ciudad española de Granada.

\section{APROXIMACIÓN TEÓRICA}

\section{El inmigrante como otro y la necesidad de un acerca- miento mutuo: la propuesta de la Educación para el Desarrollo}

Ya hemos planteado cómo las migraciones pueden constituir un importante foco de diversidad, lo que implica que se construya la figura del inmigrante, el extranjero, como un gran otro, lejano, diferente y des- conocido. Sin embargo, a su vez, debido a la globalización y sus prácticas transnacionales, el extranjero se halla cada vez más próximo (Cortés, 2008), pero sin que se dé un verdadero intercambio y acercamiento con la sociedad receptora. Es por ello que se torna interesante plantear iniciativas que busquen, precisamente, el fomento de la convivencia, y que la focalicen, conjuntamente con la integración ${ }^{2}$, como dos procesos que deben darse de forma bidireccional: tanto autóctonos como extranjeros deben integrarse en los nuevos panoramas sociales de creciente diversidad, deben estar abiertos al acercamiento y conocimiento del otro. Pero, ¿cómo lograr esto si, en realidad, lo imperante suele ser la mera coexistencia, con más o menos éxito, sin que se dé una verdadera convivencia favorecida por la interculturalidad y el mutuo enriquecimiento?

Una de las propuestas de la cooperación española se ha centrado en la Educación para el Desarrollo -en adelante, EpD-. Desde la Agencia Española de Cooperación Internacional para el Desarrollo (AECID) ${ }^{3}$, la $\mathrm{EpD}$ se define como un proceso educativo -formal, no formal e informal-, encaminado a promover una ciudadanía global generadora de una cultura de la solidaridad comprometida con la lucha contra la pobreza y la exclusión y la promoción del desarrollo humano y sostenible. En este sentido, se afirma que de nada sirven los esfuerzos por cambiar el sur sin un compromiso de llevar a cabo una transformación del norte, donde se originan y mantienen gran parte de estos desequilibrios.

La EpD, aunque es planteada desde la cooperación estatal, es implementada mayoritariamente por la cooperación descentralizada y formulada desde las Comunidades Autónomas (CCAA). Para éstas, esta estrategia constituye una de sus herramientas principales. Las CCAA, además de no tener competencias en materia de gestión migratoria (Cortés, 2004), centran su actuación de forma más próxima a fenómenos como la integración y la convivencia entre migrantes y autóctonos. Así, las propuestas de EpD abogan por el fomento del mutuo acercamiento y la integración mediante acciones que den a conocer la realidad migratoria y las experiencias de las personas migrantes, y sensibilicen sobre las relaciones desiguales entre estados emisores y receptores. Este tipo de acciones

2 Al igual que ocurre con la noción de desarrollo, el término integración será adoptado desde una postura crítica, tal y como se expondrá más adelante.

3 La definición propuesta desde la AECID para la EpD se halla disponible en su página web. Recuperada de http://www.aecid.es/ES/la-aecid/ educaci\%C3\%B3n-y-sensibilizaci\%C3\%B3n-para-el-desarrollo [último acceso octubre de 2020]. 
deberían conllevar una mayor contextualización de la complejidad del fenómeno migratorio, así como del propio concepto de desarrollo, favoreciendo el fomento de una ciudadanía implicada y concienciada con estas realidades (Aragall, 2010).

Por otra parte, en algunos proyectos de EpD -como el analizado en este texto-, son las personas migrantes las que adoptan el rol de educadores y educadoras, de agentes de desarrollo, asumiendo la tarea de dar a conocer su realidad migratoria. Para llevar a cabo su labor, los migrantes son previamente formados como agentes de desarrollo, trabajando de forma conjunta con la ONGD en que se amparan dichos proyectos. Que asuman este rol favorece que la migración y el desarrollo se vinculen del modo positivo planteado desde el codesarrollo (Sanmartín, 2010), el cual también les otorga un rol protagónico en los proyectos y acciones de desarrollo de y en sus lugares de origen. Sin embargo, pese a la bidireccionalidad y beneficio mutuo entre sociedades emisoras y receptores promulgados desde el codesarrollo (Crespo, 2015), no siempre se tienen en cuenta las posibles aportaciones que pueda tener el fenómeno migratorio en las segundas, más allá del beneficio económico derivado del trabajo de quienes migran. No se plantean las posibles contribuciones culturales de la presencia de personas extranjeras. Como afirma Crespo, (2015: S28/71) «la reciprocidad es la base del co-desarrollo, para ello es necesario superar la visión actual del inmigrante africano asociada a la pobreza económica y también cultural; cuya única aportación reconocida es su condición de mano de obra barata, contribuyente fiscal y demográfico». Con estas acciones de EpD, busca abandonar la idea del migrante como el gran otro, lejano y desconocido, favoreciendo así el acercamiento y la mejora en la convivencia que este conlleva. Desde esta perspectiva, el vínculo entre migraciones y desarrollo y las acciones en las que este es trabajado en el ámbito de la integración, se tornan relevantes para la Antropología, haciendo hincapié en el interés etnográfico hacia las aportaciones socioculturales que las personas migrantes hacen a y desde la EpD a las sociedades receptoras.

\section{EL CODESARROLLO Y SU CONCEPTUALIZACIÓN COMO HERRAMIENTA DE INTEGRACIÓN}

El codesarrollo se basa principalmente en el vínculo positivo entre fenómeno migratorio y desarrollo, entendiendo que ese sentido positivo se refiere a las aportaciones que podrían tener las migraciones actuales en el incremento de los niveles de desarrollo y la disminución de la pobreza de los lugares de los que provienen o entre los que se mueven los migrantes, así como con los efectos que este incremento del desarrollo podría tener, a su vez, en el comportamiento de los flujos migratorios (Sanmartín, 2010). Aunque las acciones y prácticas a las que alude el codesarrollo han sido llevadas a cabo, de un modo u otro, por parte de las personas migrantes desde mucho antes de la institucionalización del término, éste se popularizó como tal en el contexto francés a finales de los 90 del siglo pasado: en 1997 Sami Naïr publicó un informe centrado en esta materia, lanzando una propuesta para integrar inmigración y desarrollo, vinculando también los Estados de origen y destino de los flujos migratorios, de modo que ambos pudieran beneficiarse (Naïr, 1997).

Asimismo, en su propuesta los y las migrantes tenían un papel fundamental en la consecución de estos objetivos comunes, para la cual se marcaba como eje principal la regulación de los flujos migratorios (Naïr, 1997). En España, el codesarrollo aparece estrechamente vinculado al planteamiento francés y a la política migratoria europea implementada desde el Consejo Europeo de Tampere (Cortés, 2004), y se plantea muy unido a la política de Cooperación Internacional para el Desarrollo (Giménez et al., 2006; Lacomba y Cloquell, 2017). Es más, en el contexto español surge en un primer momento vinculado a la cooperación descentralizada ${ }^{4}$, para luego ser institucionalizado en la política estatal de Cooperación Internacional para el Desarrollo (Pinyol y Rollo, 2010). La primera década de los 2000 constituyó el momento de mayor auge del codesarrollo en España, ocupando un lugar relevante en las políticas tanto migratorias como de Cooperación Internacional para el Desarrollo. Sin embargo, desde entonces se ha perdido el interés por el mismo, desapareciendo como tal de muchos de los documentos oficiales de ambas políticas, lo que resulta relevante si tenemos en cuenta que, por el contrario, el debate sobre el vínculo migración-desarrollo sigue estando a la orden del día (Lacomba y Cloquell, 2017). También se vio reducida la cantidad de investigaciones dedicadas al mismo, pese a que en los últimos años ha resurgido un cierto interés por esta noción, vinculada sobre todo a la promoción de un desarroIlo sostenible. En este sentido, siguiendo a Lacomba y Royo (2020), el codesarrollo puede volver a tener

4 Destaca como pionera la cooperación realizada en el contexto catalán, clave en la definición e implementación de proyectos de codesarrollo. 
relevancia en el debate político actual, en el sentido en que la Agenda 2030 y sus objetivos de Desarrollo Sostenible (ODS) o los recientes pactos migratorios ${ }^{5}$ vuelven a destacar el papel de las personas migrantes y sus organizaciones en los procesos de desarrollo.

Por otra parte, el codesarrollo se centra también en el vínculo establecido entre las sociedades de origen y las receptoras, en que los migrantes se erigen como puente transnacional entre ambas (Lacomba, 2010). Cobra aquí un papel relevante la denominada sociedad civil migrante (Lacomba y Royo, 2020) como potencial promotora de un desarrollo compartido pues, a diferencia de la cooperación internacional, desde el codesarrollo se aboga en mayor medida por la bidireccionalidad y horizontalidad entre los Estados implicados (Giménez et al., 2006). Esta reciprocidad forma parte, entre otras cosas, de su carácter innovador como forma de gestionar la realidad migratoria (Crespo, 2002). En este sentido, este beneficio compartido se establece a partir de la combinación de dos tipos de acciones: aquellas vinculadas al desarrollo de los lugares de origen, juntamente con acciones de construcción de ciudadanía en sociedades receptoras (Aragall, 2010), ocupando en todas ellas un papel esencial los y las migrantes. En un principio, el interés por las acciones de desarrollo que conlleva el codesarrollo solía, y suele centrarse, en el envío de remesas por parte de los y las migrantes (Lotero-Echeverri y Pérez-Rodríguez; 2019), haciendo que, como propone Cortés (2004), no solamente interese la gestión migratoria, sino también la gestión de capitales.

Principalmente, el codesarrollo, sobre todo en su discurso institucional estatal, se ha centrado en el retorno (Naïr, 1997) y la regulación e incluso prevención de los flujos migratorios (Marín, 2006), a través de diferentes acciones de externalización de las fronteras y control migratorio, por un lado, e implementación de acciones de desarrollo en origen que fijen a los potenciales migrantes frenando así la emigración, por el otro (Malgesini, 2001). El caso es que, mayoritariamente, el desarrollo ha sido concebido y definido únicamente en su vertiente económica, entendiéndose como sinónimo de crecimiento económico: los Estados en vías de desarrollo lograrán estar desarrollados cuando alcancen los niveles de crecimiento económico de los Estados considerados ya desarrollados, modelo a seguir por los primeros en esta concepción lineal y unidireccional del concepto ${ }^{6}$. Para lograr este objetivo, se emplean la cooperación y la Ayuda Oficial al Desarrollo (AOD) derivada de la misma, en un intento por reducir la pobreza de los países del sur global $y$, en el caso que nos ocupa, entendiendo a su vez que este crecimiento económico y el desarrollo resultante frenará también la necesidad de emigrar. Asimismo, estos procesos pueden conllevar también una homogeneización cultural, una destrucción de la diferencia (Escobar, 1999): al adoptar la cultura occidental imperante, se facilita el desarrollo económico y el ingreso de estos Estados en el sistema mundial de intercambio de bienes y servicios, para lo cual deberán renunciar a sus propias formas culturales, aproximándose a la asimilación como modelo de integración. A esta visión del desarrollo como crecimiento económico se adapta también el codesarrollo, bajo la premisa de que, a más desarrollo, menos migraciones (Malgesini, 2001). Es decir, la cooperación internacional se emplearía como una herramienta más en la gestión migratoria, tal y como se concibe desde las sociedades del norte, donantes de AOD a la vez que de receptoras de flujos migratorios.

En este sentido, si en materia de codesarrollo la mayoría de acciones adoptadas se sitúan en las sociedades emisoras, pese a ser planteadas y formuladas desde las receptoras, quedaría pendiente plantearse de qué forma, o formas, puede llevarse a cabo un verdadero codesarrollo en estas últimas, con tal de dar respuesta a una de las premisas principales del fenómeno del codesarrollo: que ambas se vean beneficiadas por las migraciones y por las acciones de quienes migran (Crespo, 2015). De este modo, autores como Lacroix (2009) o el propio Naïr (2008), entre otros, lo plantearon también como una oportunidad para fomentar la integración de las personas migrantes en dichas sociedades, favoreciendo la interculturalidad y la convivencia: entra en juego la concepción del codesarrollo como herramienta de integración, de forma que tanto migrantes como población autóctona se vean beneficiados. Crespo (2015) denomina a esta dimensión relacional, dialogal y de cooperación activa como codesarrollo intercultural. Sin embargo, no podemos caer en el error de plantear a la población en términos binarios, migrantes -otros- por un lado y autóctonos -nosotros- por el otro, considerando que sólo los primeros deben integrarse. Este tipo de discursos en términos dicotómicos no hace más que

5 Podemos resaltar el Pacto Mundial para una Migración Segura, Ordenada y Regular, adoptado en Marrakech en 2018.

6 Respecto a esta cuestión, resulta interesante aproximarse al planteamiento crítico a la noción de desarrollo planteado por autores como Escobar (1999). 
perpetuar los estereotipos y el desconocimiento, causantes, entre otros, de la falta de convivencia intercultural (Vallés, 2017).

En esta misma línea, otra cuestión que parece interesante plantear se refiere, precisamente, al fenómeno de la integración. ¿Qué entendemos por integración? ¿Quién debe integrarse y de qué modo? Como decimos, la integración no debería ser considerada como una mera adaptación por parte de los migrantes, abandonando sus realidades culturales previas, sino que estas deberían servir también como nuevos modelos de los que pueda aprender la sociedad receptora (Crespo, 2004). Asimismo,

«la gestión de la incorporación de personas de diversos orígenes étnicos y nacionales a una sociedad resulta crucial para construir un mínimo denominador común en cuanto a valores y prácticas socialmente reconocidas y aceptadas, y constituye un aspecto nuclear en el proceso de integración» (Moncusí y Albert, 2013: 41).

Pero, en definitiva, podemos considerar que el concepto de integración, al igual que ocurre con el de desarrollo e incluso el de codesarrollo, debe ser empleado con cautela, siendo analizado desde una perspectiva crítica, dados los efectos que pueden tener en la vida de todas las personas implicadas en procesos de movilidad e incorporación en las sociedades plurales.

Con todo esto, para poder ser realmente de codesarrollo, las acciones puestas en marcha deben implicar a, y llevarse a cabo en, las sociedades receptoras. Debe haber una actuación en destino, en que se aprenda sobre y desde el fenómeno migratorio (Crespo, 2004) fortaleciendo la participación y concienciación de la ciudadanía. Se pretende así dar el salto de la mera coexistencia multicultural imperante, a una verdadera convivencia intercultural, y para ello el codesarrollo, en su perspectiva intercultural, puede ser de gran utilidad. Cabe destacar aquí que, siguiendo a Giménez (2003), entendemos tanto la multiculturalidad como la interculturalidad como dos modalidades diferentes del pluralismo cultural que caracteriza a las sociedades actuales. La interculturalidad vendría a ser una alternativa necesaria frente a las limitaciones de la multiculturalidad, entendida como mera yuxtaposición de personas culturalmente diversas, sin que se dé una verdadera interacción entre ellas -cosa que sí ocurriría en el caso de la primera- (Giménez, 2003). Asimismo, la interculturalidad, según Crespo (2002), favorece el intercambio mutuo sin renunciar a la identidad de las partes en contacto, pese a que todavía no podamos dar por superado el asimilacionismo intrínseco de algunos modelos de integración (Giménez, 2003; Crespo, 2015). Y como hemos anunciado, en este tipo de acciones e iniciativas juegan un papel esencial quienes migran, erigiéndose como agentes de desarrollo, aquí y allí, en procesos de desarrollo y de integración. Además, dado que todas estas cuestiones han venido siendo relevantes para la Antropología, el codesarrollo, al incorporarlas y vincularlas a la cuestión migratoria y su impacto en el desarrollo compartido, también pasa a convertirse en una temática de interés para la misma.

En las sociedades receptoras, estas formas de codesarrollo pueden ser concretadas en acciones de EpD, tal y como se expone a continuación, por lo que las personas migrantes dejan de ser considerados como meras beneficiarias de la cooperación para pasar a adoptar un rol activo y participativo en ambas sociedades. Desde esta perspectiva, que considera el codesarrollo como una combinación de desarrollo en origen e integración en destino (Pérez del Olmo, 2009), éste nos permitiría llevar a cabo acciones en las sociedades receptoras de cara a mejorar la convivencia y el mutuo entendimiento, que beneficiarán en igual medida a migrantes y población local. Así,

«la información y la sensibilización y educación para el desarrollo son instrumentos que permiten enfocar las migraciones como un fenómeno social positivo, no exento de retos y dificultades, pero cargado de oportunidades. En este sentido, una información veraz y objetiva sobre la realidad migratoria en origen y en destino contribuiría positivamente a transformar la percepción social del fenómeno migratorio» (Pérez del Olmo, 2009: 69).

Con todo esto, consideramos interesante que el codesarrollo deje de ser entendido únicamente como una herramienta de control de flujos migratorios -objetivo que, cabe señalar, es perseguido por las administraciones y que no tiene por qué ser compartido por las personas migrantes-, y que, en su vertiente como herramienta de cooperación al desarrollo, conlleve también acciones en las sociedades receptoras. Éstas obtienen múltiples beneficios del fenómeno migratorio, tanto a nivel económico (Daum, 2004), como social y cultural: siguiendo a Graciela Malgesini, «el contacto entre culturas que se genera a partir de la presencia de inmigrantes tiende a generar efectos mutuamente enriquecedores" (Malgesini, 2001: 145). Pero, si tiene lugar en las sociedades receptoras, no solo las personas migrantes deben participar en sus 
acciones: si nos centramos en el codesarrollo como herramienta de integración (Lacroix, 2009), sí puede -o debería- verse implicada la población autóctona.

\section{APUNTE METODOLÓGICO}

Para llevar a cabo nuestra investigación, analizamos un caso concreto empleando un acercamiento etnográfico implementando diferentes técnicas de carácter cualitativo, centrales en Antropología: observación participante de varias fases y actividades del proyecto, entrevistas semi-estructuradas con migrantes africanos participantes en el proyecto, y análisis documental de documentos vinculados al mismo -formulación del proyecto, informes intermedios y finales, encuestas realizadas a los diferentes beneficiarios del proyecto, etc.-. Esta multiplicidad de técnicas nos ha permitido acceder a material variado y diverso, cuya información obtenida fue triangulada de cara a la obtención de nuestros resultados de investigación (Valles, 1999).

\section{Presentación del proyecto analizado}

El proyecto a partir del cual se construye esta reflexión fue formulado y llevado a cabo por la ONGD Alianza por la Solidaridad. Hasta el momento se han llevado a cabo dos ediciones; la primera, finalizada en 2015 y que tuvo lugar en las ciudades de Granada, Huelva y Sevilla; y la segunda, realizada a lo largo del curso 2017/2018 en Granada y Sevilla, y en ambos casos fue financiado mayoritariamente por la Agencia Andaluza de Cooperación Internacional para el Desarrollo (AACID) ${ }^{7}$. El título general del proyecto fue Andalucía se llena de África. La primera edición se subtituló Africanos y africanas acercan el continente mostrando su sabiduría, riqueza, ritmos y sabores, y la segunda Reflexión, debate y construcción de herramientas para el fortalecimiento del colectivo africano como agente de transformación.

Ya desde los títulos podemos intuir los objetivos del proyecto: que las personas migrantes de África ${ }^{8}$ den a conocer sus conocimientos acerca de su continente, lugares de origen ${ }^{9}$ y realidad migratoria. El objetivo general del proyecto en su primera edición fue «sensibilizar a la sociedad andaluza sobre la potencialidad de la población de África y la corresponsabilidad del norte en su empobrecimiento»; y el objetivo específico número uno se concretó en «sensibilizar a la población andaluza, a las instituciones y ONGD sobre África a través de acciones protagonizadas por africanos y africanas formados como agentes de educación para el desarrollo» ${ }^{10}$. Con todo ello, a partir del proyecto se pretendía, primero, sensibilizar a la población andaluza sobre la migración africana y sus protagonistas; segundo, formar a las personas migrantes para que fueran ellas mismas las educadoras y sensibilizaran a la población local; y tercero, fomentar espacios de encuentro, intercambio y aprendizaje mutuo entre andaluces y africanos. Los migrantes africanos tuvieron un papel protagónico en todas las fases del proyecto, y fueron formados como agentes de EpD, diseñando e implementando diferentes acciones (charlas, talleres, actividades de intercambio) para dar a conocer su visión, percepciones y vivencias sobre la migración africana y la convivencia en y con la sociedad andaluza.

A continuación, vamos a centrarnos en analizar el rol que puede tener la EpD en la gestión de la convivencia y formas de integración entre ambas poblaciones, la africana y la autóctona, así como el vínculo entre migraciones, cooperación para el desarrollo y acciones llevadas a cabo en la sociedad receptora, desde un planteamiento vinculado a la emergencia del codesarrollo en nuestro contexto.

\section{RESULTADOS Y DISCUSIÓN}

\section{Migrantes como agentes de Educación para el Desa- rrollo}

Siguiendo a Almudena Cortés, veíamos como «bajo el paraguas del codesarrollo se refuerzan mutuamente las categorías discursivas que presentan un "Tercer Mundo subdesarrollado" que ahora, además, es "migrante". Es más, es porque procede de un contexto

7 El análisis aquí presentado ha sido realizado mayoritariamente en base a la primera edición que tuvo lugar en la ciudad de Granada, pese a que el mismo ha sido complementado y enriquecido a partir de la participación en algunas actividades de la segunda edición.

8 El proyecto estaba centrado en la región del sur del Sáhara, descartando el norte del continente africano, por lo que, cuando nos referimos a africanos y africanas, en este caso, excluimos a las personas procedentes de estados del África septentrional. Por otro lado, nos llama la atención la propuesta de Crespo (2015), de denominar a esta población africanos «sudsaharianos», en lugar del habitual «subsaharianos", por las connotaciones negativas que puede tener el prefijo sub.

9 Cabe destacar que la mayoría de los y las participantes eran de origen senegalés, pues esta constituye la nacionalidad de África al sur del Sáhara con mayor representación en Granada, por lo que su presencia fue mayor también en el proyecto.

10 Tanto los objetivos como la información técnica del curso fueron obtenidos del documento de Formulación de Proyectos de Educación para el Desarrollo, presentado por la Fundación CEAR - Habitáfrica en 2011 (documento no publicado). 
subdesarrollado por lo que emigra» (Cortés, 2008: 54). Sin embargo, y, por otra parte, hemos comprobado también cómo, desde el codesarrollo, emerge la idea del migrante como vector de desarrollo, agente activo del mismo, con la delegación de responsabilidades en materia de desarrollo local que esto puede implicar. Esto, como afirma Cortés (2008), supone una gran contradicción en la construcción de la figura del migrante en y desde el codesarrollo, vinculado sobre todo al fenómeno de la cooperación, pues los y las migrantes ocupan una doble posición como agentes y como beneficiarios/as de las ayudas derivadas de la cooperación. Y, es más, en muchos casos, se diseñan los proyectos para ellos/as, pero sin contar con sus aportaciones y visiones (Cortés, 2008). Esta forma de representar al migrante refleja una tensión derivada también de la idea del extranjero como otro, lejano, distante, desconocido, diferente y ajeno a nosotros.

En este desafío de buscar alternativas adecuadas en la forma que representamos al otro, la Antropología puede jugar un papel esencial, al incorporar en sus reflexiones la forma que tienen los propios migrantes de representarse y generar conocimientos sobre sí mismos (Cortés, 2008). Y, en todo ello, tienen especial cabida y relevancia las acciones en que se busque dar a conocer su realidad, sensibilizando a la población autóctona sobre el fenómeno migratorio y la situación de las personas migrantes tanto en los procesos migratorios como en los de incorporación en las sociedades que se autodenominan de acogida. Estas actuaciones "se pueden enmarcar en la educación para el desarrollo y la concientización de la población receptora sobre los movimientos migratorios y sus aportaciones al desarrollo del conjunto de la sociedad" (Crespo, 2015: S28/71). Así, a través de este tipo de iniciativas, en que se incluyen aquellas consideradas de EpD, se busca un acercamiento con la población autóctona que permita superar el desconocimiento, sin perder de vista el hecho de que este esfuerzo debe ser bidireccional y no solo adoptado por parte de las personas migrantes.

Como afirma Crespo «la perspectiva intercultural aplicada al codesarrollo favorece relaciones equitativas en las que las personas de la sociedad receptora se encuentran al mismo nivel respecto a las personas migradas porque no conocen las sociedades de origen ni el funcionamiento de los procesos migratorios» (Crespo, 2015: S28/71). De este modo será posible lograr la reciprocidad preconizada por el codesarrollo, para lo cual es necesario superar la visión del inmi- grante -de origen africano en este caso-, asociada a la pobreza económica y cultural (Crespo, 2004). La inmigración africana debe ser considerada en sus múltiples dimensiones, como fuente de enriquecimiento y aprendizaje. Y, para ello, ¿quién mejor que las propias personas migrantes africanas, que han vivido todos estos procesos, para darlos a conocer en las sociedades receptoras? Además, si el conocimiento etnográfico está en el núcleo de la disciplina antropológica, el que este sea proporcionado por sus protagonistas, desde una perspectiva emic, acerca la EpD en este formato a los intereses antropológicos. De este modo, el proyecto analizado nos resultó de interés como antropólogas precisamente por el modo en que es trabajado y presentado el conocimiento, desde un planteamiento próximo a las Epistemologías del Sur, en que se habla del continente africano en toda su diversidad y desde las voces de las propias personas africanas.

En este sentido, entra aquí en juego la importancia del reconocimiento de la agencia y autonomía de las personas migrantes, de escuchar su voz, así como su capacidad por aportar a las sociedades receptoras en -supuesta- igualdad de condiciones. A través de la difusión de sus propias trayectorias migrantes, sus percepciones y vivencias respecto a estas y a los procesos de incorporación en las sociedades receptoras, las personas migrantes pueden participar de la transformación social que se persigue desde las acciones de educación para la ciudadanía global. Su implicación en proyectos de EpD -entendida como una rama más de la propia cooperación para el desarrollo-, va en beneficio de la construcción de sociedades plurales que valoren la diversidad cultural y aboguen por modelos de inserción más basados en la interacción, el intercambio y el enriquecimiento mutuo, que en la mera yuxtaposición de personas.

\section{Educación para el Desarrollo, codesarrollo y partici- pación}

En base a lo planteado hasta el momento, podemos desarrollar una serie de cuestiones respecto a la forma en que se relacionan la EpD y el codesarrollo a través de las acciones implementadas por los y las migrantes, centrándonos en el proyecto Andalucía se llena de África. La puesta en práctica de acciones de este tipo, que persiguen dar a conocer la realidad de las personas africanas residentes en las ciudades andaluzas, así como los procesos migratorios vividos por éstas, busca fomentar el acercamiento y la mejora en las relaciones, partiendo de la premisa de que es 
precisamente la falta de conocimiento mutuo lo que impide la comunicación y, con ello, la convivencia. Podríamos afirmar que, si el codesarrollo se planteaba como vínculo entre sociedades de origen y sociedades receptoras, de modo que ambas se vean beneficiadas, es a través de acciones de EpD que pueden obtenerse resultados positivos en las segundas, más allá de las meras contribuciones económicas que puedan hacer quienes migran en su rol de trabajadores y trabajadoras. Es decir, el fenómeno migratorio debe ser considerado desde una visión que ponga de relieve sus beneficios, como fuente de enriquecimiento cultural y social, en que las personas migrantes vean reconocida su voz y su visión, la cual sirva para favorecer la construcción de una ciudadanía activa, participativa y abierta a la convivencia con el otro. De todos modos, su participación en proyectos de este tipo debe ser analizada desde una perspectiva crítica, teniendo en cuenta varias cuestiones.

Primero, pese a que las personas migrantes son las protagonistas del proyecto, podemos cuestionarnos algunos aspectos relacionados con la formación de estas como agentes de desarrollo: quién educa a quién, desde qué punto de vista, con qué objetivos y en base a qué conocimientos previos. Pese a que los migrantes acaben adoptando un rol protagónico en estas acciones, han sido formados previamente por las ONGD o docentes de las universidades de la sociedad receptora, siempre desde una postura próxima y abierta al fenómeno migratorio. Además, podemos plantearnos hasta qué punto se puede lograr esa horizontalidad buscada y propulsada por el codesarrollo, si las personas intervinientes no parten, desde un principio, de la misma posición, ni poseen -o se les permite- la misma capacidad de agencia.

Por otra parte, reflexionamos también sobre quiénes se benefician del proyecto, $y$, asimismo, quiénes participan y por qué. Por un lado, muchos de los migrantes ya se habían implicado previamente en acciones de este tipo, con la misma ONGD o por su cuenta, $y$, además, formaban parte del tejido asociativo de la ciudad. De este modo, ¿es adecuado reflexionar sobre la participación en codesarrollo de migrantes si aquellas que acaban participando son personas que parecen estar ya más concienciadas acerca de la pertinencia de todas estas acciones o de implicarse en actividades de sensibilización y convivencia? ¿Cuáles son los intereses que les mueven a todo ello? ¿Tienen el mismo rol activo y participante en acciones en sus lugares de origen?
Lo mismo ocurre con la población andaluza que acude a este tipo de actividades. Es cierto que las sesiones en escuelas y universidad pueden venir impuestas por quienes enseñan, pero también suelen atraer a personas que vean necesarias este tipo de acciones. Ocurre lo mismo con quienes asistentes a charlas, talleres y actividades libres ofertadas. De este modo, podemos cuestionarnos hasta qué punto son adecuadas las actividades de sensibilización si, al final, acaban llegando a un tipo concreto de población, en cierto sentido ya concienciada e implicada en estos procesos de participación y construcción de ciudadanía. Así, podemos plantearnos qué personas acaban acercándose y sintiéndose atraídas por este tipo de acciones y por qué, y cuáles, por el contrario, no lo hacen. Con todo ello, podemos cuestionarnos si, finalmente, es la sensibilización la práctica más adecuada para el objetivo de mejorar la convivencia.

En cuanto al impacto de este tipo de proyectos, si partimos de una perspectiva transnacional (Faist, 2010), para mejorar la vida de las personas migrantes debemos trabajar centrándonos en los sistemas que vinculan el allí y el aquí, pues el desarrollo en origen no servirá de nada si no se mejoran las condiciones de vida y la convivencia en las sociedades receptoras, y viceversa. Al fin y al cabo, la migración es un fenómeno que no va a detenerse y que probablemente siga incrementándose, haciendo que las ya de por sí sociedades plurales y heterogéneas lo sigan siendo (Lotero-Echeverri y Pérez-Rodríguez, 2019). Por este motivo, parece adecuado seguir llevando a cabo acciones que favorezcan no solo la convivencia, sino también las relaciones transnacionales entre Estados, caracterizadas hoy en día por un gran desequilibrio. En esta línea, este proyecto adopta una visión mucho más local (o translocal), centrada en la vida de quienes migran en la sociedad receptora y en la convivencia con la población autóctona, buscando que se desdibuje el límite entre ambos colectivos y tenga lugar un verdadero acercamiento entre ellos. También se persigue superar el desconocimiento mutuo basado en estereotipos y prejuicios fomentados, a su vez, por los medios de comunicación y algunas autoridades políticas (Vallés, 2017). Un conocimiento renovado sobre el continente africano, que ponga de relieve su enorme diversidad sociocultural, que refuerce la idea de que África no es un país, mostrando experiencias concretas trasmitidas por las personas africanas. Este enfoque local se halla estrechamente relacionado, como ya anunciábamos, con el fenómeno de descentralización de la cooperación en España: acciones locales más vinculadas 
a la integración y la acogida de las personas migrantes que a la gestión y control migratorios.

Con todo ello, los participantes valoraron muy positivamente este proyecto, recalcando la posibilidad de acercarse a la población autóctona desde una posición de horizontalidad desde la que poder aportar sus conocimientos, percepciones y sentimientos sobre el fenómeno migratorio. Este intercambio y aprendizaje mutuos parece beneficiar la convivencia intercultural, acercándonos a la propuesta de Giménez (2003) de una inclusión basada en la aceptación de la diversidad cultural como un aspecto social positivo. En este sentido, pese a que quienes participaron afirmaron que, en la mayoría de los casos, sus relaciones con las personas locales no eran negativas, en realidad esa convivencia se caracterizaba, básicamente, por la ausencia de relaciones, es decir, por una mera coexistencia, causada, según expresaron, por el mutuo desconocimiento (Vallés, 2017): no conocer el continente africano, su diversidad social y cultural y su larga historia fomenta la construcción de la figura del africano como otro, desconocido, lejano a la vez que próximo, y percibido únicamente en su condición de inmigrante.

Asimismo, además del intercambio y acercamiento con la población andaluza, destacaron el haber podido crear y mantener el contacto con otras personas migrantes residentes en Granada, tanto de su misma nacionalidad como de otras procedencias, implicadas todas ellas en este tipo de iniciativas. Ello les permitió establecer una red para futuras colaboraciones en acciones de sensibilización, concienciación y, en definitiva, intercambio con la sociedad receptora, que es ahora también la sociedad en que desarrollan su vida cotidiana. Esta creación de redes entre personas migrantes y/o asociaciones de migrantes fue subrayada también como uno de los aspectos más positivos del proyecto. Esto nos hace destacar la importancia de un tejido asociativo fuerte entre migrantes, que dé amparo a su participación social como ciudadanía activa. Como afirma Mourad Aboussi «el asociacionismo inmigrante constituye una clara prueba del dinamismo y la ciudadanía activa, aunque la estructura de oportunidades no favorezca su emergencia y visibilidad dentro de la sociedad receptora» (Aboussi, 2015: S28/8). Pero, entonces, ¿qué ocurre con los migrantes que quedan fuera de este tejido asociativo? ¿No participan a nivel social? ¿O lo hacen de modo diferente? ¿Cómo pueden implicarse en acciones colectivas y de creación de ciudadanía? ¿Qué entendemos, con todo ello, por participar? Resulta interesante seguir reflexionando sobre estas cuestiones a partir de proyectos implementados en las sociedades receptoras, pero que tengan a las personas migrantes como actores protagonistas además de beneficiarios.

\section{A MODO DE CONCLUSIÓN, REGRESO A LA AN- TROPOLOGÍA}

Con todo lo expuesto hasta el momento, y desde nuestra posición como antropólogas, seguimos con más dudas que posibles respuestas. Queríamos plantear este trabajo de forma que nos permitiera reflexionar sobre cómo la Antropología, desde el enfoque de los estudios migratorios, puede aproximarse a los fenómenos de codesarrollo y EpD, aportando una visión más compleja, completa y crítica, aplicable a este tipo de proyectos. En este sentido, y siguiendo a Cortés (2008), desde una orientación pública y comprometida, la Antropología se interesa por el codesarrollo por sus implicaciones en la vida de las personas migrantes, y nos interpela a nosotras mismas, a nuestra responsabilidad social como miembros de una comunidad determinada. Desde la Antropología como ciencia participamos en la construcción de formas de representar al otro. Y si, como veíamos, esa construcción de alteridades constituye un tema de gran interés y uno de los motivos por el que puede interesarnos el fenómeno migratorio (Vallés, 2017), el codesarrollo, en cuanto hace precisamente esto, también nos interesa. Pero, al trabajar conjuntamente con la EpD, la construcción del -ya no tan- otro podría acercarse más a las propias formas de autorrepresentarse de los y las migrantes, además de abrir una puerta y aproximarse cada vez más a los conocimientos y aprendizajes de los que podemos beneficiarnos desde culturas diversas y distantes (Cortés, 2008).

Así, este tipo de proyectos pueden verse muy beneficiados por el conocimiento antropológico en sus múltiples vertientes, por ejemplo, la teoría transnacional de las migraciones, su perspectiva intercultural, y la denominada Antropología del desarrollo, desde la que, como ya anunciábamos, se ha venido criticando el propio concepto de desarrollo (Escobar, 1999). Desde esta perspectiva se intenta establecer una visión que supere la concepción de esta noción como proceso económico, lineal, y que apueste por incorporar nuevos saberes y modos de hacer las cosas y de desarrollarse. Este ámbito de la Antropología, así, emerge como campo específico de estudio en el que pueden tener cabida los estudios destinados al fenómeno migratorio, en cuanto el vínculo migración-desarrollo 
sigue teniendo enorme relevancia social y política, y para el cual se necesitan enfoques críticos como el ya descrito. La Antropología puede también aportar su granito de arena «dando a conocer realidades que parecen enfrentadas, al interesarse precisamente por estos migrantes como sujetos de otredad, que ayudan a construir los propios procesos de identificación de los “locales" »(Vallés, 2017: 2316). Y, del mismo modo, debe reconsiderar su posición en la configuración del codesarrollo en el campo transnacional, no solo en la sociedad receptora, sino también en los lugares de origen y tránsito, lo que afectará a su vez a la forma en que, como investigadoras, llevemos a cabo nuestro propio trabajo de campo.

En cuanto al codesarrollo, parece pertinente seguir concretando el modo en que este puede tomar forma en las sociedades receptoras, dando respuesta a su definición como proceso que vincula, implica y tiene repercusiones en las sociedades que se ven atravesadas por el fenómeno migratorio. En este sentido, desde la gran crisis económica de los últimos años, las subvenciones recibidas por la cooperación al desarrollo se han visto reducidas y esta reducción ha ocupado un lugar poco destacado en los debates políticos. Sin embargo, el vínculo con el fenómeno migratorio sigue siendo relevante, por lo que parece adecuado buscar otras formas de poner sobre la mesa el codesarrollo, esta vez más vinculado a las acciones en las sociedades receptoras. De este modo, el codesarrollo como herramienta de integración o de acercamiento con el otro, puede servir de altavoz a las personas migrantes, favoreciendo la convivencia y modificando los procesos de construcción de identidades basados en la alteridad de la figura del migrante. En definitiva, la Antropología puede aportar una visión más compleja, holística y crítica de todos estos fenómenos, desde la que seguir reflexionando sobre cuál puede ser su papel cuando las migraciones se vinculan con el desarrollo y la integración, y qué implicaciones puede tener en la configuración del codesarrollo en estos contextos trasnacionales de relaciones interculturales. Además, podemos preguntarnos también qué puede aportar esta disciplina a la propia Cooperación Internacional para el Desarrollo, la cual, como ocurría con las demás nociones planteadas, podría verse beneficiada de un proceso de revisión y replanteamiento desde nuevas perspectivas, más abiertas a la horizontalidad y el enriquecimiento mutuos.

\section{REFERENCIAS}

Aboussi, Mourad (2015). Codesarrollo institucional versus codesarrollo académico: enfoques preventivos, politizados e idealistas de una idea con potenciales de desarrollo compartido. En García Castaño, Francisco Javier; Megías, Adelaida y Ortega, Jennifer (eds.). Actas del VIII Congreso sobre Migraciones Internacionales en España. Granada: Instituto de Migraciones, pp. S28/3-S28/10.

Aragall, Xavier (2010). I Congreso Internacional de Codesarrollo: Conclusiones. En: Solé, Carlota (coord.) Migraciones y desarrollo. El codesarrollo: del discurso a la práctica. Barcelona: Anthropos, pp. 271-283.

Cloquell, Alexis (2013). Migración y desarrollo. El vínculo del codesarrollo. Valencia: Editorial Tirant Lo Blanch.

Cortés, Almudena (2004). La emergencia del codesarrollo: una emergencia transnacional. Actas del IV Congreso sobre la inmigración en España: ciudadanía y participación (10-13 de noviembre), pp. 915-930.

Cortés, Almudena (2008). Antropólog@s en el codesarrollo: opciones para un análisis desde el compromiso público de la Antropología. En: Jabardo, Mercedes; Monreal Pilar y Palenzuela, Pablo (coords.) Antropología de orientación pública: visibilización y compromiso de la antropología. San Sebastián: Ankulegi, pp. 43-60.

Crespo, Rafael (2002). Una visión intercultural de las condiciones, condicionantes y riesgos en torno al desarrollo. En: Subirà, Nuria (ed.). Codesarrollo: planteamientos y perspectivas. Barcelona: Edicions La Terra, pp. 62-69.

Crespo, Rafael (2004). Codesarrollo, un nuevo concepto para una antigua realidad. Actas del IV Congreso sobre la inmigración en España: ciudadanía y participación (10-13 de noviembre), pp. 931-944.

Crespo, Rafael (2015). Más de una década de codesarrollo, ¿qué hemos aprendido? La visión de un Tubab. Propuesta de análisis desde la perspectiva intercultural. En García Castaño, Francisco Javier; Megías, Adelaida y Ortega, Jennifer (eds.). Actas del VIII Congreso sobre Migraciones Internacionales en España. Granada: Instituto de Migraciones, pp. S28/63-S28/74.
Daum, Christophe (2004). Migrantes, protagonistas del desarrollo: una mediación de dos espacios. Mugak, 27-28, pp. 7-12.

Escobar, Arturo (1999). Antropología y Desarrollo. Maguaré, 14, pp. 42-73.

Faist, Thomas (2010). Transnationalization and Development: Toward and Alternative Agenda. En: Glick-Schiller, Nina y Faist, Thomas (eds.) Migration, Development and Transnationalization. EEUU: Berghahn Books, pp. 63-99.

Giménez, Carlos (2003). Pluralismo, multiculturalismo e interculturalidad. Propuesta de clarificación y apuntes educativos. Educación y futuro: revista de investigación aplicada y experiencias educativas, 8 , pp. 11-20.

Giménez, Carlos, Martínez, Julio L., Fernández, Mercedes y Cortés, Almudena (2006). El codesarrollo en España. Protagonistas, discursos y experiencias. Madrid: La Catarata.

Grosfoguel, Ramón (2006). La descolonización de la economía política y los estudios postcoloniales: transmodernidad, pensamiento fronterizo y colonialidad global. Tabula Rasa, 4, pp. 17-46. 
Lacomba, Joan (2010). El codesarrollo: debilidades y fortalezas de una idea y su práctica. En: Solé, Carla (coord.) Migraciones y desarrollo. El codesarrollo: del discurso a la práctica. Barcelona: Anthropos, pp. 37-57.

Lacomba, Joan y Cloquell, Alexis (2017). Políticas de desarrollo en el contexto de la migración. Coherencias y contradicciones en el caso de dos programas de codesarrollo de España en Colombia y Ecuador. Iberoamerican Journal of Development Studies, 6 (1), pp. 50-74.

Lacomba, Joan y Royo, Isabel (2020). Los ODS y la sociedad civil migrante. EI encaje de las asociaciones de inmigrantes en las políticas de cooperación al desarrollo en España. Iberoamerican Journal of Development Studies, 9(1), pp. 232-257. doi: 10.26754/ojs_ried/ ijds. 458

Lacroix, Thomas. (2009). Migrations, Développement, Codéveloppement: quels acteurs pour quels discours? [en línea]. [Fecha de consulta: 16 de octubre de 2020]. Disponible en: https://halshs. archives-ouvertes.fr/halshs-00820369/ document

Lotero-Echeverri, Gabriel y Pérez-Rodríguez, M. Amor (2019). Migraciones en la sociedad contemporánea: Correlación entre migración y desarrollo. Retos. Revista de Ciencias de la Administración y Economía, 9(17), pp. 145-159. https:// doi.org/10.17163/ret.n17.2019.09
Malgesini, Graciela (2001). Reflexiones sobre migraciones, cooperación y codesarrollo. Arxius de Ciències Socials, 5, pp. 123-146.

Marín, Isabel (2006). La cooperación española para el desarrollo como prevención de la emigración marroquí: percepciones, discursos y realidades entre dos orillas. [Tesis doctoral inédita]. Universidad de Granada: Granada.

Moncusí, Albert y Albert, María (2013). El rol del asociacionismo. De inmigrantes africanos en la construcción de la cohesión social y la convivencia en Cataluña, Navarra y la Comunidad Valenciana. Miradas cruzadas. Revista Internacional de Sociología (RIS). Monográfico sobre asociacionismo e inmigración, 71, pp. 39-65.

Naïr, Sami (1997). Informe de balance y orientación de la política de codesarrollo en relación con los flujos migratorios. Misión Interministerial sobre Migración y Codesarrollo. Ministerio de Asuntos Exteriores de Francia.

Naïr, Sami (2008). Codéveloppement et flux migratoires. Migrations Société, 3 (117-118), pp. 71-76. https://doi. org/10.3917/migra.117.0071

Pérez del Olmo, Fernando (2009). 100 cuestiones básicas sobre migraciones y codesarrollo. Castilla La Mancha: Asociación para el Desarrollo de la Manchuela y Asociación para el Desarrollo Integral Mancha Júcar - Centro.
Pinyol, Gemma y Royo, Enric (2010). El concepto de codesarrollo en las políticas públicas: una historia inconclusa. En: Solé, Carlota (coord.) Migraciones y desarrollo. El codesarrollo: del discurso a la práctica. Barcelona: Anthropos, pp. 83-123.

Sanmartín, Anna (2010). El codesarrollo en España: un análisis de la implicación de los migrantes. [Tesis doctoral inédita]. Universidad Complutense de Madrid: Madrid.

Valles, Miguel S. (1999). Técnicas cualitativas de investigación social. Reflexión metodológica y práctica profesional. Madrid: Editorial Síntesis.

Vallés, Alba (2017). Cómo el otro se descubre como tal: discursos y percepciones de migrantes senegaleses sobre la construcción de su identidad en el contexto migratorio. En: Vicente, Teresa; García, Pepa; Vizcaíno. Antonio (eds.) Antropologías en transformación: sentidos, compromisos y utopías. Valencia: Universitat de València, pp. 2308-2317.

Zamora, José Antonio (2003). Inmigración, ciudadanía y multiculturalidad. En: Zamora, J.A. (coord.) Ciudadanía, multiculturalidad e inmigración. Navarra: Editorial Verbo Divino, pp. 165-240.

Zapata, Ricard (2003). La ciudadanía en contextos de multiculturalidad. Procesos de cambios de paradigmas. Anales de la Cátedra Francisco Suárez, 37, pp. 173-200. 